\title{
Enhancing surface reactivity of boron nitride nano ribbon by chlorine gas decoration: A computational study
}

\author{
Kiran Kumar Surthi ${ }^{1}$, and Kamal K. Kar ${ }^{1,2^{*}}$ \\ Advanced Nanoengineering Materials Laboratory \\ ${ }^{1}$ Materials Science Programme and ${ }^{2}$ Department of Mechanical Engineering, Indian Institute of \\ Technology Kanpur, Kanpur, Uttar Pradesh-208016, India. \\ *Email: kamalkk@iitk.ac.in
}

First principle calculations have been carried out to investigate the surface reactivity of chlorine gas terminated zigzag boron nitride nano ribbon (ZBNNR $\left.{ }_{\mathrm{Cl} 2 \mathrm{~B}-\mathrm{Cl} 2 \mathrm{~N}}\right)$ within the density functional theory (DFT) by using local density approximation (LDA). In $\mathrm{ZBNNR}_{\mathrm{Cl} 2 \mathrm{~B}-}$ $\mathrm{Cl}_{2} \mathrm{~N}, \mathrm{Cl}_{2}$ induces electrons into the conduction band, as a result their consequent electronic states across the Fermi level enhance the electrical conductivity. Charge density analysis reveals that the chlorine gases introduce excess negative charge into the active surface of ZBNNR that brings an enhancement in the surface reactivity.

Boron $\left(2 p^{1}\right)$ and nitrogen $\left(2 p^{3}\right)$ are neighbors of carbon; the electronic structure of boron nitride (BN) is identical to the carbon $\left(2 \mathrm{~s}^{2} 2 \mathrm{p}^{2}\right)$. It demonstrates structural polymorphs that are analogous to carbon polymorphs and exhibiting properties including large surface area, wide energy gap, chemically and thermally inert, etc. [1]. One dimensional (1D) thick strip from 2D $\mathrm{h}-\mathrm{BN}$, it is termed as BNNR.

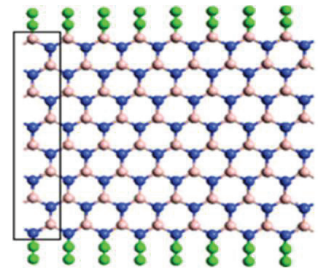

Figure 1: ZBNNR $\mathrm{Cl}_{2 \mathrm{~B}-\mathrm{Cl} 2 \mathrm{~N}}$ (blue, brown, white, and green spheres are $\mathrm{N}, \mathrm{B}, \mathrm{H}$ and $\mathrm{Cl}$ respectively)

To investigate the charge density analysis of both edge $\mathrm{Cl}_{2}$-terminated ZBNNRs. We apply the first principles calculations based on the linear combination of atomic DFT method implemented in the atomistic tool kit (ATK) [2]. Which is completely on real space, non equilibrium Green function's formalism. We have employed local density approximation (LDA) as proposed by Perdew. et al. [3]. For periodic boundary condition of ZBNNRs model along z-axis, other two dimensions are confined (Figure 1). We have selected an energy cut-off of 100 Rydberg and the k-point sampling is set to $1 \times 1 \times 100$. Considered structure is optimized with no constraint until forces on atoms are less than $0.05 \mathrm{eV} / \AA$. The optimized configuration with average bond length values of B-N, B-H and N-H are 1.46, 1.1 , and $1.06 \AA$ respectively.

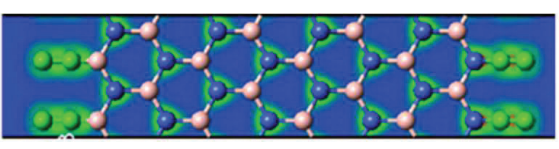

Figure 2: Charge density of iso-surface of $\mathrm{ZBNNR}_{\mathrm{Cl} 2 \mathrm{~B}-\mathrm{Cl} 2 \mathrm{~N}}$

At B-edge, 4s-orbital with unpaired electrons of $\mathrm{Cl}$ is bonded by $\sigma$-bonding with $2 \mathrm{~s}$ (sp2hybridized)-orbital of B-atom. At N-end, unpaired $2 p_{x}$-orbital of $\mathrm{N}$ is bonded by $\sigma$-bonding with $4 \mathrm{~s}$-orbital of $\mathrm{Cl}$. As a result, $3 \mathrm{p}_{\mathrm{y}}$ orbital of $\mathrm{Cl}$ with single electron on both sides of the ribbon is available for conduction [4].

Charge density iso-surface of $\mathrm{ZBNNR}_{\mathrm{C1} 2 \mathrm{~N}-\mathrm{C} 12 \mathrm{~B}}$ is depicted in Figure 2. Charge transfer from $\mathrm{Cl}$ to $\mathrm{N}$-atom is $0.126 \mathrm{e}$. Therefore, the valence charge density for highest valance band (VB) is 0.10 and it is predominantly accountable on N$\mathrm{Cl}$ bond at $\mathrm{N}$-edge. For conduction band (CB), the charge density assessment is 0.164 and charge transfer from $\mathrm{Cl}$ to $\mathrm{B}$ is $-0.04 \mathrm{e}$. Hence, $\mathrm{Cl}_{2}$-gas introduces negative charge in active surface of the ribbon leads to enhancement of negative charge availability throughout the ribbon surface and predicting $\mathrm{Cl}_{2}$-gas sensing properties of ZBNNRs.

\section{References}

1. J. Yu, Z. Zhang, W. Guo, J. Appl. Phys. 113 (2013) 133701.

2. M. Brandbyge, J. -L. Mozos, P. Ordejón, J. Taylor, K. Stokbro, Phy. Rev. B. 65 (2002) 165.

3. J. P. Perdew, A. Zunger, Phy. Rev. B.23 (1981) 10.

4. B. Huang, Z. Li, Z. Liu, G. Zhou, S. Hao, J. Wu, B. L. Gu, W. J. Duan, Phys. Chem. C 112 (2008) 13442. 